\title{
Are spatial and dimensional attention separate? Evidence from Posner, Stroop, and Eriksen tasks
}

\author{
ERAN ChaJuT \\ The Open University of Israel, Ra'anana, Israel \\ AND \\ Asi Schupak and Daniel Algom \\ Tel-Aviv University, Tel-Aviv, Israel
}

\begin{abstract}
Do various operational definitions of visual attention tap the same underlying process? To address this question, we probed visual selective attention using orientation of attention, flanker, and Stroop tasks. These were embedded in combined designs that enabled assessment of each effect, as well as their interaction. For the orientation task, performance was poorer at unexpected than at expected locations. The flanker effects also differed across the two locations. In contrast, the Stroop effects were comparable at expected and unexpected locations. We conclude that spatial attention (tapped by the orientation and the flanker tasks) and dimensional attention (tapped by the Stroop task) engage separate processes of visual selection, both of which are needed in normal attention processing.
\end{abstract}

Selective attention enables the prioritization of resources for processing the most salient and consequential portions of visual input. Are these portions regions or objects in space (hence the need for spatial attention) or attributes of a single object (hence the need for dimensional attention)? Both possibilities have been considered in the literature, without much crosstalk between the pertinent bodies of research. The notion of visual field or space is vital in the first approach. Attention parses the visual field (initially) into well-processed and less well-processed chunks, the parsing governed by physical proximity (as space-based models hold) or by shared figural, temporal, or other physical features (as object-based models hold). The divide between space-based accounts and objectbased accounts notwithstanding, it is important to realize that, in this approach, attention always operates on chunks of the input, and, hence, in this basic sense, it can be said to be "holistic" (cf. Goldsmith, 1998; Shalev \& Algom, 2000). The notion of a "spotlight" oriented through attention (Posner, Snyder, \& Davidson, 1980) has come to epitomize much - though not all—of the pertinent research.

In the second approach, by contrast, attention is an intraobject process, decomposing the stimulus into taskrelevant and task-irrelevant dimensions. Because the relevant dimension must be abstracted from the whole stimulus, attention is said to be "analytic" in this approach. Note that the notion of object- or space-based selection is gratuitous in the second approach because a single object is considered (hence the absence of object-based selection) whose attributes often inhere in the same location (hence the absence of space-based selection). Attention to the word or to the color attributes of Stroop stimuli exemplifies this approach.

Are the two species of selective attention related or independent? Are both required for normal attention? If so, what is their modus operandi? In order to answer these questions, in this study we combined the two classes of attention into a single experimental design. Because Posner's orientation task (Posner, 1978, 1980; Posner \& Raichle, 1994; Posner \& Rothbart, 2004; Posner et al., 1980) taps spatial attention, whereas the Stroop (1935) task taps analytic intraobject attention, we expected an additive outcome when conjoining the respective manipulations. We also used a third task, the flanker task introduced by C. W. Eriksen and Hoffman (1973; see also B. A. Eriksen \& Eriksen, 1974), in which the observer makes a speeded response to a central letter while attempting to ignore nearby lettersflankers - that are or are not associated with the required response. Because the Posner and the flanker tasks both entail a significant spatial component, we expected the respective manipulations to interact. Together, the PosnerStroop combination, on one hand, and the Posner-flanker combination, on the other hand, enable a principled dissociation of the two classes of selective attention.

Posner's orientating task, on one hand, and the Stroop task, on the other hand, map onto different attentional systems. The orienting of attention entails a process of selecting one out of several sources of information separated from one another in space (whether as regions or as objects). Note that this process does not involve the logical relation of conflict or agreement, but merely that of alignment or disengagement of attention from the target. The

E. Chajut, eranch@openu.ac.il 
Stroop task, by contrast, applies to objects whose constituent dimensions stand in conflict or agreement with one another. Therefore, selective attention in this task seeks to decompose the object into its constituent dimensions, focusing on the task-relevant one. Commensurate with the disparate nature of the two systems of attention are the variables that have been tested and shown to affect each. Chief among the variables affecting the orientation of attention is spatial separation of the objects or their components. Chief among the variables affecting the Stroop effect is the relative salience of the dimensions. Because the Stroop task entails fine-grained intraobject analysis (typically involving conflict), it is widely considered a prime process of executive attention (Botvinick, Carter, Braver, Barch, \& Cohen, 2001).

The present analysis is compatible with the work done by Posner and his associates on networks of attention (Fan, McCandliss, Fossella, Flombaum, \& Posner, 2005; Posner \& Petersen, 1990; Posner \& Raichle, 1994). Thus, "orienting and executive control are widely thought to be relatively independent aspects of attention that are linked to separable brain regions" (Fan et al., 2005, p. 471). Earlier, Posner and Raichle selected the orienting and Stroop tasks as those most directly engaging the spatial and executive control systems, respectively. Despite this work on attention networks tests, the networks and the associated tasks have not been sufficiently segregated. Often, the relevant experiments yielded interaction rather than the expected independence (e.g., Fan, McCandliss, Sommer, Raz, \& Posner, 2002). The use of the flanker task to stand for the executive conflict resolution network proved a major source of confusion.

The crucial involvement of spatial factors in the flanker task has been ignored. This task actually engages the spatial system (much like the orienting task does). In this study, we show that the orienting and the flanker tasks interact in a predictable manner, the interaction attributable to the shared spatial system of attention. The response conflict or agreement in the flanker task is modulated by advance spatial cuing of the stimuli (rendering them expected or unexpected) precisely because this task shares resources with the orienting-spatial system.

Therefore, the unique contributions of this study are threefold. First, we performed a double dissociation of representative tasks of orientation and Stroop, thereby demonstrating the full independence of the underlying systems for the first time. Second, we clarified the role of the two modes of attention through the flanker task, which involves components from both networks. Third, viewed from a wider perspective, in this study, we addressed the apparent anomaly by which two conceptions of selectivity that go by a common name and speak to kindred issues lack dedicated attempts at integration.

\section{Three Measures of the Selectivity of Attention: Posner, Stroop, and Eriksen Effects}

Spatial attention: Posner's orientation of attention task. The first effect derived in the present experiments was Posner's advance spatial cuing or orientation effect (Posner, 1978, 1980; Posner \& Raichle, 1994; Posner \& Rothbart, 2004; Posner et al., 1980). The effect is measured via the orientation-of-attention paradigm: The participant is asked to detect a target (usually, a simple signal) that can appear at several locations in the visual field (typically, at the left or right periphery). Advance information on the likely location of the peripheral target is provided by a brief cue. This cue helps to orient attention in space-hence the designation of the paradigm (cf. Folk, Remington, \& Johnston, 1992; Posner \& Cohen, 1984). The cue faithfully indicates the future location of the target on some trials (valid trials), but it is misleading on other trials (invalid trials). The effect of attention is gauged by the difference in performance on invalid and valid trials.

Dimensional attention: The Stroop task. The second effect derived in the present study was the Stroop effect. The Stroop (1935) task is widely used to measure attention in the analytic approach. The paradigm and the associated effect are well known. A single color word in color is presented on a trial, and the participant's task is to name, while timed, the print color. The stimuli divide into congruent (the word naming its color as in the word RED printed in red) and incongruent (word and color conflict as in RED printed in green) combinations. The Stroop effect is the difference in performance across congruent and incongruent combinations. ${ }^{1}$ The presence of the Stroop effect shows that the task-irrelevant words were noticed, thereby compromising exclusive focusing on the target color. The absence of the Stroop effect, on the other hand, documents good selective attention to the target print colors. Because the defining feature of the Stroop task is the presence of congruent and incongruent combinations, the effect can be assessed using a large variety of stimuli (see MacLeod, 1991, and Melara \& Algom, 2003, for reviews). A ready example is the hierarchical letters popularized by Navon (1977), in which a large letter is composed of many (identical) small letters. We used these stimuli in the present study.

Navon's (1977) stimuli satisfy the basic Stroop stipulation, because all possible combinations of large and small letters yield congruent (same large and small letters) or incongruent (different letters) combinations. Indeed, hierarchical letters have been popular stimuli in the Stroop literature (Kinchla, Solis-Macias, \& Hoffman, 1983; Martin, 1979; Pomerantz, 1983; Ward, 1982). With the original Stroop stimuli, a reverse effect is sometimes observed, by which print color intrudes on word reading (Stroop, 1935; see Algom, Dekel, \& Pansky, 1996, and Melara \& Mounts, 1993, for recent examples). With hierarchical letters, the direction of the effect is more uniform, with the large letter typically intruding on performance with the small letters but not vice versa (global precedence).

Spatial attention to parts: The flanker task. The third effect derived in the present study was the flanker effect (B. A. Eriksen \& Eriksen, 1974; B. A. Eriksen, Eriksen, \& Hoffman, 1986; C. W. Eriksen \& Hoffman, 1973; Lachter, Forster, \& Ruthruff, 2004). In the typical flanker task, a triplet of letters is presented, and the participants give one response if the central target letter belongs to one category (e.g., an A or $\mathrm{H}$ ) and give another response if it belongs to another category (e.g., an S or C). The flanker effect is the finding that responses are faster on trials in which the flanking letters are assigned the same response 
as the target letter than on trials in which they are assigned the opposite response.

The response-compatibility effect in the flanker task is somewhat analogous to the Stroop effect, but, unlike the Stroop effect, it depends crucially on the spatial separation of the components. The flanker effect is markedly reduced - to the point of elimination - when the distance between the flankers and the target is increased $\left(1^{\circ}\right.$ of visual angle is a convenient limit; see C. W. Eriksen \& St. James, 1986). This trivial fact betrays the spatial quality of the flanker task, a quality that is missing from the Stroop task. In the Stroop task, the dimensions inhere in the same location; hence, spatial attention does not play a role.

\section{The Double Attention Paradigm}

The double attention paradigm (DAP) entails the concurrent manipulation of two attention effects within a single experiment. The paradigm allows the derivation of both effects on the basis of the single speeded response measured. We applied three species of the DAP in the present study.

DAP I: Posner and Stroop effects in a single experiment. We combined the manipulation of presenting the target stimulus in expected and unexpected locations (Posner) with the manipulation of presenting congruent (same large and small letters) and incongruent (different letters) target stimuli (Stroop). The observer was asked to respond to just one attribute (say, small letters) while ignoring the other attribute, which corresponded to or conflicted with the relevant attribute. Following Posner, a brief cue preceded the target, which appeared in the periphery for a limited time in validly cued or in invalidly cued locations. Following Stroop, the target was not a simple signal, but rather a Stroop stimulus of a large letter composed of many small letters. Hence, the required response is not simply detection but, rather, also involves identification or classification of the target attribute (small letters or the large letter).

The two effects are readily derived. Because the stimulus appears at expected and unexpected locations, the Posner effect is given by the difference in performance across the two classes of trials. Because the stimuli appear in congruent and incongruent preparations, the difference in responding to the target attribute across the two combinations provided the Stroop effect. The focal contrast of interest concerned the Stroop effects observed in valid and invalid trials. Comparable effects would imply independence of spatial/holistic and intraobject/analytic attention, whereas differential effects would implicate a common system (cf. Fan et al., 2002; Posner \& Petersen, 1990; Posner \& Raichle, 1994).

In an earlier DAP study, Shalev and Algom (2000) found an effect of orientation of attention (better performance at expected than at unexpected locations), as well as the Stroop effect (better color-naming performance for congruent than for incongruent stimuli). Most important, comparable amounts of the Stroop effect obtained at spatially attended or expected (validly cued) and spatially unattended or unexpected (miscued) stimuli. Shalev and Algom concluded that input selection from the visual field and dimensional selection from the stimulus engage separate processes of attention.
However, caution is invited before drawing strong conclusions. For one example, Chen (2003) reported larger Stroop interference at expected than at unexpected locations in some (although not all) conditions of a study aimed at testing selectivity of attention under load. ${ }^{2} \mathrm{Al}-$ though Chen's interest was focused on the effects of load on attention, one must be circumspect, given the conflicting reports.

Given the key issue of the equivalence of the Stroop effects, we created a more sensitive measure of their magnitude by presenting hierarchical letters. With these Stroop stimuli, the effect is almost exclusively directional (the large letter intruding on the small ones), so that the interference is fully concentrated in one dimension (with colors and words, one often gets reverse Stroop effects, too).

Finally, the need for caution also comes from the fact that it is risky to draw strong inferences from the absence of a difference in the respective Stroop effects (i.e., from a null effect). Double dissociation of the spatial and analytical modes of attention can alleviate this concern. This was accomplished in the present study by applying a second DAP, this time combining the Posner and flanker tasks. We expected differential flanker effects in expected and unexpected locations - in sharp contrast to the hypothesis of comparable Stroop effects - because of the shared spatial component of the orientation and flanker tasks.

DAP II: Posner and flanker effects in a single experiment. The procedure for the Posner task was the same as that used in DAP I. Following the standard flanker task, the stimuli were triplets of letters in which the central one required a response that was the same as or the opposite from that for the irrelevant flankers. The three letters appeared at the periphery, preceded by a cue informative of their likely (left or right) location. The participants' task was speeded classification of the central letter. The effect of orientation or expectance was calculated as before, the difference in performance between validly and invalidly cued stimuli. The flanker effect was calculated as the difference in performance between response-congruent and responseincongruent triplets.

Recent DAP studies combining the Posner and the flanker tasks reported that they interact. Capitalizing on the design developed by Shalev and Algom (2000), Fan et al. (2002) found that the Posner and the flanker effects interact; the interaction was inconsistent with their hypothesis of independent networks of attention (cf. Posner \& Petersen, 1990; Posner \& Raichle, 1994). Similar results were reported by Lupiáñez and his associates (Callejas, Lupiáñez, \& Tudela, 2004; Lupiáñez \& Funes, 2005) and by Danziger, Kingstone, and Ward (2001). Consequently, we expected different amounts of the flanker effect at validly cued, or expected, and miscued, or unexpected, locations. Moreover, we hypothesize that the flanker effect will be larger at invalidly cued than at validly cued locations. The prediction is grounded in the unique nature of the flanker task. This task involves a conflict or agreement between spatially separated components (the Stroop task involves conflict or agreement between spatially integrated components). Inevitably, the spatial mode of attention is invited, a mode that segregates in a fine-grained manner the visual 
input at the attended region. This segregation attenuates the effect of the flankers (whether congruent or incongruent) at the cued location.

DAP III: Posner and color Stroop effects in spatially integrated and spatially separated preparation. In a final DAP design, we used the original Stroop stimuli of color words printed in various ink colors. This standard spatially integrated preparation was complemented by a spatially separated display in which the color word in black was accompanied by a colored bar at a neighboring location (cf. Kahneman \& Chajczyk, 1983). The observer responded to the color while ignoring the word (whether integrated or separated). A brief cue appeared in the periphery, indicating (for valid trials) or falsely indicating (for invalid trials) the subsequent location of the Stroop stimulus.

The effect of orienting was calculated as the difference between the generic speed of responding to validly cued and that to invalidly cued trials. The Stroop effect was calculated as the difference in RT between congruent and incongruent stimuli. As we did before, we calculated the Stroop effect separately for validly cued and invalidly cued trials. Our interest was focused on the following two contrasts. The first concerned the presence or absence of a difference in the Stroop effects observed across validly cued or spatially attended, and invalidly cued or spatially unattended, locations (see the DAP I section). The second concerned the presence or absence of a difference between the Stroop effects with spatially integrated and spatially separated stimuli (see DAP II section).

Our predictions were as follows. On the basis of the earlier results by Shalev and Algom (2000), we predicted comparable Stroop effects at spatially attended or expected, and at spatially unattended or unexpected, locations with the original Stroop stimuli. We thus predicted that the results of DAP I and DAP III would converge on the same pattern of invariant Stroop effects, providing strong evidence for the separability of spatial and analytic modes of attention. The predictions differed with respect to the spatially separated Stroop stimuli. Because these stimuli engage spatial attention, different amounts of Stroop effects are expected at validly cued or expected, and invalidly cued or unexpected, locations. On the basis of the same considerations discussed with respect to the flanker effect, we predicted larger Stroop effects at spatially unattended or unexpected locations. For these stimuli, we expected that the results of DAP II and DAP III would converge, thereby providing complementary evidence on the separability of the two systems of attention.

In summary, we juxtaposed spatial and dimensional modes of selective attention in Experiment 1, using Posner's and Stroop's tasks, respectively. We combined two attention effects with a significant spatial component in Experiment 2, using Posner's and C. W. Eriksen and Hoffman's (1973) tasks, respectively. In Experiments 3 and 4, we combined the Posner and the Stroop tasks using the original Stroop stimuli of color words and colors. In these experiments, we directly contrasted the effects obtained with or without spatial separation of components. Again, separability in Experiment 1 versus interaction in Experi- ment 2 supports the sought dissociation of spatial/holistic and intraobject/analytic modes of attention. In particular, the direct contrast performed in Experiments 3 and 4 provides decisive evidence on the modus operandi of the two systems.

\section{EXPERIMENT 1 \\ The Orientation of Attention to Components of Stroop Stimuli}

\section{Method}

Participants. Fourteen Open University undergraduates took part in the experiment in partial fulfillment of a course requirement. All of the participants had normal or corrected-to-normal visual acuity, as was assessed by self-report. Their age ranged from 20 to 38 years.

Stimuli, Apparatus, and Design. The target stimuli were compound letters closely patterned after those used and illustrated by Martin (1979, Figure 1) and by Pomerantz (1983, Figure 1). A large (global) letter ( $\mathrm{H}$ or $\mathrm{S}$ ) was formed by the appropriate arrangement of many small (local) letters (H or S). Two of the resulting stimuli contained the same letter at both local and global levels (congruent combinations), whereas the other two contained different letters (incongruent combinations). The stimuli were generated by a Dell Pentium computer and displayed in white on the black background on a 17 -in. color monitor with an $85-\mathrm{Hz}$ refresh rate and set at a resolution of $1,024 \times 768$ pixels. The participants were seated at a viewing distance of approximately $60 \mathrm{~cm}$ from the screen, such that each global letter subtended a visual angle of $3.6^{\circ} \times 2.1^{\circ}$, whereas that of the local letters was $22 \times 14 \mathrm{~min}$ of arc. The stimuli appeared to the left or to the right of the center of the screen with their inner sides located $2.6^{\circ}$ of visual angle from fixation. The advance cue consisted of a small filled circle and was presented at the peripheral side of one of the possible locations. The circle subtended a visual angle of $0.5^{\circ}$ in diameter and was presented $5.2^{\circ}$ to the right or left of fixation. The advance cue and the subsequent target stimulus (when on the same side) were separated by $0.5^{\circ}$, measured from the outer edge of the target.

Each trial entailed the following sequence. A "+" sign was presented at fixation for $1 \mathrm{sec}$, followed by a 40 -msec blank screen. Then the peripheral cue appeared for $100 \mathrm{msec}$, followed by a 40-msec blank screen, at the end of which appeared the target for $50 \mathrm{msec}$. An interval of $0.5 \mathrm{sec}$ followed each response. A block included 120 trials, $75 \%$ of which were attended (preceded by a valid adjacent cue) and $25 \%$ unattended (preceded by an invalid remote cue). Each block started with 12 trials for training (unbeknownst to the participants). Of each type of stimuli, half were presented to the right of fixation and half were presented to the left of fixation. The various trials - attended and unattended - were intermixed in a random fashion in the block, subject to the provision that no more than 2 unattended trials or 4 trials with the same response appeared in a sequence.

Procedure. The participants were tested individually in a dimly lit room. Each participant performed in two separate blocks, one with the local letter as the relevant dimension and the other with the global letter as the relevant dimension. A break of 10 min separated the two experimental blocks. Speeded classifications of $\mathrm{H}$ or $\mathrm{S}$ were made by pressing one or the other of two vertically aligned central keys. The order of blocks (local versus global classification) and key assignment were counterbalanced across participants.

\section{Results}

Global letter as the target attribute revealed a robust effect of spatial attention, yielding faster (by $43 \mathrm{msec}$ ) and more accurate (by 2.65\%) responses at expected than at unexpected locations [for reaction time (RT), $M=449$ and $492 \mathrm{msec}$, respectively, $F(1,13)=30.8, p<.0001$; for error, $M=4.6 \%$ and $7.25 \%$, respectively, $F(1,13)=2.06$, 
$p=.17]$. In contrast, Stroop effects were absent at both expected and unexpected locations [for RT, $M=445$ and $452 \mathrm{msec}$ for congruent and incongruent stimuli, respectively, at attended locations, and $M=488$ and $496 \mathrm{msec}$ for congruent and incongruent stimuli, respectively, at unattended locations; for error, $M=4.4 \%$ and $4.8 \%$ for congruent and incongruent stimuli, respectively, at attended locations, and $M=5.8 \%$ and $8.7 \%$ for congruent and incongruent stimuli, respectively, at unattended locations; $F<1$, for all comparisons]. Our participants classified global letters that were composed of mismatching local letters as speedily and accurately as they did global letters composed of matching local letters. Invalid cuing exacted a toll on performance, but stimulus composition did not. The global letters were free of interference from conflicting local letters and did not reap gain from matching local letters. The insularity of the global letters is not surprising, because we purposely created the compound letters with enhanced salience of the global dimension.

Responses to the local letters were slower by $223 \mathrm{msec}$ than those to global letters $[F(1,13)=88.3, p<.0001]$. Errors were also more numerous for local than for global letters by $8.48 \%$ on average $[F(1,13)=22.6, p<.0005]$. This is the well-known global precedence documented for compound letters (in which the global attribute is more salient than the local one). The detailed results with respect to the local letters are presented in the left-hand panel of Figure 1. Evident in Figure 1 is the effect of spatial cuing, with better overall performance at expected than at unexpected locations. The difference favoring stimuli at attended locations amounted to $145 \mathrm{msec}[F(1,13)=$

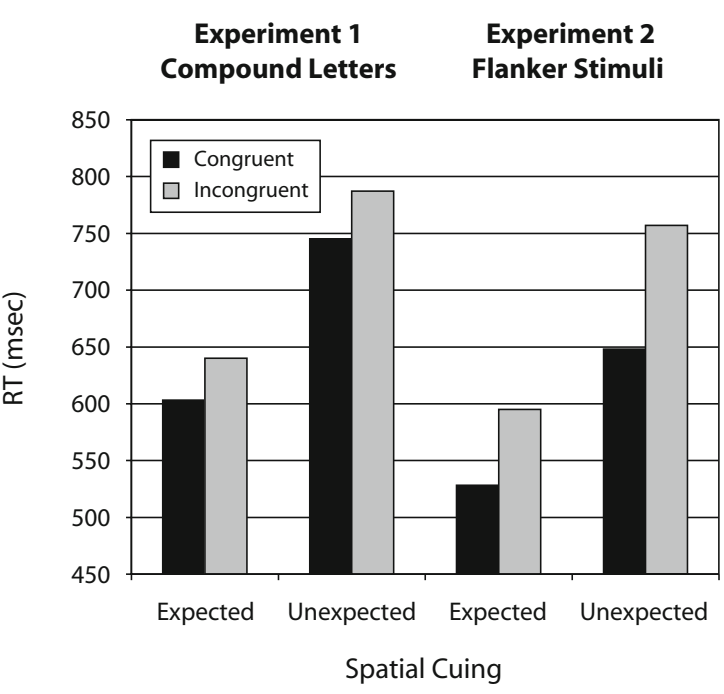

Figure 1. Posner and Stroop effects (Experiment 1) and Posner and flanker effects (Experiment 2) for classifications of (local) letters. The differences in reaction time (RT) between expected and unexpected locations document the effects of spatial orientation of attention. The differences in RT between congruent and incongruent stimuli at each location document Stroop and flanker effects. Note that the Stroop effects are roughly the same at the two locations, whereas the flanker effects are larger at unexpected than at expected locations.
$17.41, p<.001]$; errors were also fewer at attended than at unattended locations by $7.65 \%[F(1,13)=5.7, p<.05]$. Also evident in Figure 1 are the Stroop effects observed at both expected and unexpected locations. They amounted to $37 \mathrm{msec}$ at expected locations $[F(1,13)=21.27, p<$ $.005]$ and to $42 \mathrm{msec}$ at unexpected locations $[F(1,13)=$ $2.35, p=.07]$. The 5 -msec difference between the two Stroop effects was not reliable $(F<1)$. Errors were also fewer for congruent than for incongruent stimuli with both valid and invalid cuing, but these differences were not statistically significant $[F(1,13)=1.34, p=.267]$.

\section{Discussion}

The most striking results to emerge from this experiment are the comparable Stroop effects recorded at attended or expected, and unattended or unexpected locations. Attended stimuli engendered faster and more accurate responding than did unattended stimuli, yet congruent and incongruent stimuli instigated the same performance asymmetry with both types of stimuli. Because we purposely created compound letters with a mismatch favoring the global component, the entire Stroop effect was concentrated in the local component. These Stroop effects thus constitute very sensitive measures of irrelevant intrusion because they are not modified by (differential) reverse effects. And these sensitive measures matched in magnitude across expected and unexpected locations. We conclude that stimuli at both conditions were subjected to the same kind and strength of intrastimulus dimensional processing by the analytic system. This system acts independently from that governing cross-stimulus processing, whether space or object based. In other words, the fact that the spatial system was afforded the chance to focus on the target location did not affect the performance of the analytic system dealing with intraobject relationship.

In an auxiliary experiment done with a new group of participants, we repeated the procedures of Experiment 1 using the direction of a central arrow as the cue for orienting attention (Kuhn \& Kingstone, 2009). The same pattern of results emerged with the central arrow cue. Spatial attention was drawn successfully to the cued location, thereby improving performance at those locations. Nevertheless, the Stroop effects recorded at the two locations were virtually the same ( 36 and $35 \mathrm{msec}$ at expected and unexpected locations, respectively). The parallel results obtained with the two types of cuing show that any executive involvement with spatial attention is minimal (see Stoffer, 1993, for largely similar conclusions).

A potential concern with the results of Experiment 1 is the great asymmetry in performances observed across the global and local dimensions. Were the local stimuli, at the focus of the current interest, too difficult to detect? Were the comparable Stroop effects observed at attended and unattended locations the result of this difficulty? A close look at portions of the data allays these concerns. In point of fact, the local stimuli were highly detectable (at a level of close to $90 \%$ across the main and the auxiliary experiments). Moreover, the appreciable Stroop effects obtained with the local stimuli at both attended and unattended locations show that these stimuli were not only detected but 
also processed at a semantic level of analysis. Nevertheless, we felt the need to address this issue in a more direct fashion and did so in Experiments 3 and 4.

In order to complete the dissociation, it is desirable to show the presence of positive differences in target segregation at attended and unattended locations, if those processes are of a spatial nature themselves. This was accomplished in the next experiment, in which a flanker task replaced the Stroop task.

\section{EXPERIMENT 2 The Orientation of Attention to Components of Flanker Stimuli}

\section{Method}

Participants. A fresh sample of 14 Open University undergraduates took part in the experiment, again in partial fulfillment of a course requirement. Their ages were between 22 and 31 years.

Stimuli, Apparatus, and Procedure. The apparatus, design, location, and timing of events were the same as those in Experiment 1, but a triplet of equal-sized letters (chosen from the local letters in Experiment 1) appearing in a row replaced the compound letters of Experiment 1. The entire triplet extended $14 \mathrm{~mm}$ in width, which is slightly more than $1^{\circ}$ of visual angle when seen from the viewing distance of $60 \mathrm{~cm}$. Each letter was $4 \times 4 \mathrm{~mm}$ in size; the interletter distances were thus smaller by almost an order of magnitude than the $1^{\circ}$ separation suggested by C. W. Eriksen and St. James (1986) as the limit within which it is impossible to exclude the processing of irrelevant stimuli.

On each trial, the two letters flanking the central one were identical. The task for the participants was to classify, while timed, the central letter as $\mathrm{C}$ or $\mathrm{K}$, while ignoring the flanker letters. They had to press the top key for $\mathrm{C}$ and the bottom key for $\mathrm{K}$. The triplets $\mathrm{CCC}$ and KKK formed congruent combinations, and the triplets $\mathrm{CKC}$ and $\mathrm{KCK}$ were the incongruent combinations. Congruent and incongruent stimuli were presented equally often in both attended and unattended locations in a random fashion. Again, all of the other procedural details, including peripheral cuing and distribution of valid and invalid trials, were the same as in Experiment 1.

\section{Results}

Mean RTs are presented in the right-hand panel of Figure 1. Salient to visual inspection is the unusually great effect of cue validity. Correct classification of the central letter took $562 \mathrm{msec}$, on average, when the triplet appeared in a validly cued location, but the same classification took $703 \mathrm{msec}$, on average, when the triplet appeared in an unexpected location. This large 141-msec effect of exogenous cuing $[F(1,13)=22.2, p<.0001]$ is probably attributable to the combination of dense spacing and tight timing. The fairly demanding flanker task with stimuli presented in close proximity for a very short duration was greatly facilitated when the stimuli appeared in the expected location; commensurately, invalid cuing under these inauspicious conditions exacted a heavy toll on performance. The error data mirrored the RT data, with more errors with invalidly cued than with validly cued stimuli $[M=10.15 \%$ and $4.16 \%$, respectively, in unexpected and expected locations; $F(1,13)=8.99, p<.01]$.

For our second manipulation, appreciable flankercongruity effects were recorded at both expected and unexpected locations. For the former, the mean RTs for con- gruent and incongruent triplets were 528 and $595 \mathrm{msec}$, respectively, amounting to a flanker effect of $67 \mathrm{msec}$ $[F(1,13)=29.9, p<.0001]$. For the triplets in invalidly cued trials, the respective means were 648 and $757 \mathrm{msec}$ for congruent and incongruent triplets, respectively, amounting to a reliable effect of $109 \mathrm{msec}[F(1,13)=46.2, p<$ .0001]. Clearly, the flanker effects differed under the two conditions: The effect obtained on invalid trials was thus almost twice the value of that on valid trials $[F(1,13)=$ $4.87, p<.05$ for the interaction of stimulus type (congruent, incongruent) and cue validity (valid, invalid)]. Errors were also more numerous with incongruent than congruent stimuli in both valid and invalid locations, but neither of these trends was reliable.

Therefore, the signature of the present data is the differential flanker effects observed at attended and unattended locations.

\section{Discussion}

The Posner and the Eriksen tasks are both based on spatial filtering (on a different scale); hence, both contribute to the activation of a spatial code in arrangements such as that of Experiment 2 (see Lupiáñez \& Funes, 2005, for similar results with respect to the so-called spatial Stroop effect). A spatial code is gratuitous with Stroop stimuli, so that, in the DAP with Posner and Stroop tasks (Experiment 1), there was not an interaction of the two systems. The modulation of the flanker effect by spatial attention does not work via the banishment from perception of the flankers. If the flankers are imperceptible or less perceptible when spatial attention is focused on the central stimulus, the reduction of the flanker effect under spatially attended conditions is a fairly trivial result. However, spatial attention operates through fine-grained processing of objects in space and not by eliminating objects from space. In this respect, the evidence of Experiment 1 is instructive. Had spatial attention worked by focusing on a very small region/object at the expense of neighboring regions/ objects, a local letter would have been attended free of interference from other stimuli, including the global letter. It is for this reason that spatial attention did not make a difference in Experiment 1.

As in Experiment 1, we again added an auxiliary experiment with respect to the flanker task of Experiment 2. The cue in the auxiliary experiment was a central arrow. The results of the auxiliary experiment fully reproduced those of Experiment 2. Of most importance, the toll on performance exacted by the irrelevant flankers was larger at unexpected than at expected locations. The orienting and flanker tasks both engage the same system-hence the observed interaction.

\section{EXPERIMENT 3 The Orientation of Attention to the Color of Original Stroop Stimuli}

In Experiments 1 and 2 we accomplished a double dissociation of within-object analytic attention and betweenobjects spatial attention. When the experimental tasks 
engaged different systems, the pattern of attentional performance was additive. However, when the tasks tapped the same system, the attentional outcome was interactive.

Although this general configuration was replicated across various conditions, we encountered several methodological concerns. First, the criterial Stroop dimension used in Experiment 1 proved quite difficult. This difficulty of discriminating the local stimuli of compound letters might have contributed to the comparable Stroop effects observed at expected and unexpected locations. Second, the nature of the flanker task is not fully resolved (cf. Lachter et al., 2004), so its role as arbiter might be questioned. Third, testing on further tasks is also invited on theoretical grounds, to show that the present dissociation does not depend on the particular tasks used. Finally, the dissociation would be singularly powerful if it is demonstrated on the same task and stimuli in spatially integrated and spatially separated arrangements. This feat was attempted in the final pair of experiments with the original Stroop stimuli.

Therefore, in Experiment 3, we presented color words printed in ink colors, and the participant's task was to quickly classify the ink color, as in the standard Stroop task. Half of the stimuli were congruent (the color word named its ink color) and half incongruent (the word and the color conflicted). The classic Stroop effect was calculated as the difference in color classification performance between congruent and incongruent stimuli. The Stroop stimuli appeared at the periphery, preceded by an advance lateralized cue. On the majority of trials, the cue correctly predicted the subsequent location of the Stroop stimulus (valid trials). On the remaining trials, the cue appeared on the opposite side of the display, falsely predicting the target's location (invalid trials). Posner's orientation of attention effect was calculated as the difference in color classification performance between validly cued and invalidly cued stimuli. We tested the dependence or independence of the Stroop and Posner effects by comparing the former on valid and invalid trials.

Testing the relationship between the attention systems on the original Stroop stimuli carries several advantageous features. The task is fairly easy (under a standard preparation) and less prone to errors than that referring to components of compound letters. The task is also extremely well scrutinized. Of most importance, the same color task can be used with the word and the color separated in space (Experiment 4), thereby enabling a direct comparison of analytic and spatial modes of attention.

\section{Method}

Participants. Sixteen Open University students, undergraduates from the Department of Psychology, took part in the experiment in partial fulfillment of a course requirement. None had participated in any of the previous experiments. All had normal or correctedto-normal visual acuity, as was assessed by self-report. Their age ranged from 22 to 38 years.

Stimuli, Apparatus, and Design. The stimuli were color words (e.g., RED) printed in a congruent (red) or incongruent (e.g., blue) color. The word appeared within the black frame of one of a pair of $2 \times 2.2 \mathrm{~cm}$ (height $\times$ width) rectangles. The rectangles were pre- sented $2.6^{\circ}$ to the left and to the right of fixation (measured from the side nearer fixation). Therefore, two lateralized boxes appeared on each trial, with one containing the color-word stimulus. An advance (exogenous) cue was used with the frame of one of the boxes flickering for $50 \mathrm{msec}$. The stimulus was presented after an interval of $40 \mathrm{msec}$ (in the cued or in the noncued opposite box). Consequently, the observer had to shift spatial attention from the fixation point to the target location on each trial.

Each trial entailed the following sequence. A "+" sign at fixation along with the two boxes were presented for $1 \mathrm{sec}$. This was followed by the flickering of one of the peripheral boxes for $50 \mathrm{msec}$. After an additional $40 \mathrm{msec}$, a stimulus word appeared within one of the boxes for $200 \mathrm{msec}$. The participants' task was to respond to the ink color of the word by pressing one of a pair of vertically aligned keys. An interval of $0.5 \mathrm{sec}$ followed each response.

There were 112 trials. Of these, $75 \%$ were valid trials, in which the word appeared at the cued location; the remaining trials were invalid trials, in which the word appeared at the unexpected location. The experimental trials were preceded by 16 practice trials (unbeknownst to the participants). Of the 112 trials, half entailed congruent stimuli: The color word appeared in its matching ink color. The remaining half of the trials entailed incongruent stimuli: The word and the color conflicted. The various trials - spatially attended and unattended, congruent and incongruent - were intermixed in a random fashion subject to the proviso that no more than 2 unattended trials or 4 trials with the same response appeared in a sequence.

The stimuli were generated by a Dell Pentium computer using a 17 -in. color monitor with an $85-\mathrm{Hz}$ refresh rate and set at a resolution of $1,024 \times 768$ pixels. The participants were seated at a viewing distance of approximately $60 \mathrm{~cm}$ from the screen. Each word subtended a visual angle of approximately $1.43^{\circ}$ in width and $0.52^{\circ}$ in height.

Procedure. The participants were tested individually in a dimly lit room. They were instructed to respond to the color of the word as fast and as accurately as possible. Speeded classifications of the ink color were made by pressing one or the other of two vertically aligned keys in the center of the board. Key assignment was counterbalanced across colors and participants.

Data analysis. The error rates in this and in the following experiment were very low. In Experiment 3, the error rates for congruent and incongruent trials at expected locations were $3.2 \%$ and $3.4 \%$, respectively. The corresponding values at unexpected locations were $4.3 \%$ and $4.1 \%$. In Experiment 4, the error rates for congruent and incongruent stimuli at expected locations were $3.7 \%$ and $3.6 \%$, respectively. The corresponding values at unexpected locations were $2.9 \%$ and $2.5 \%$. None of the pertinent comparisons was statistically significant. Consequently, we do not discuss errors in these experiments. The following results are based on the correct responses.

\section{Results}

The results appear in the left-hand panel of Figure 2. Both Posner and Stroop effects are evident in Figure 2. For the former, the color responses to stimuli in expected locations ( $M=486 \mathrm{msec})$ were faster than those to stimuli in unexpected locations $(M=508 \mathrm{msec})$. The difference of $22 \mathrm{msec}$ favoring performance for spatially attended stimuli was reliable $[F(1,15)=31.7, p<.0001]$. For the latter, the participants responded to colors of congruent stimuli $(M=490 \mathrm{msec})$ faster than to those of incongruent stimuli $(M=508 \mathrm{msec})$. Of most importance for the present interest, the Stroop effects recorded at spatially attended and unattended locations were comparable. The Stroop effect for stimuli in expected locations amounted to $16 \mathrm{msec}[F(1,15)=3.77, p<.05]$, whereas that for stimuli in unexpected locations was $21 \mathrm{msec}[F(1,13)=$ 


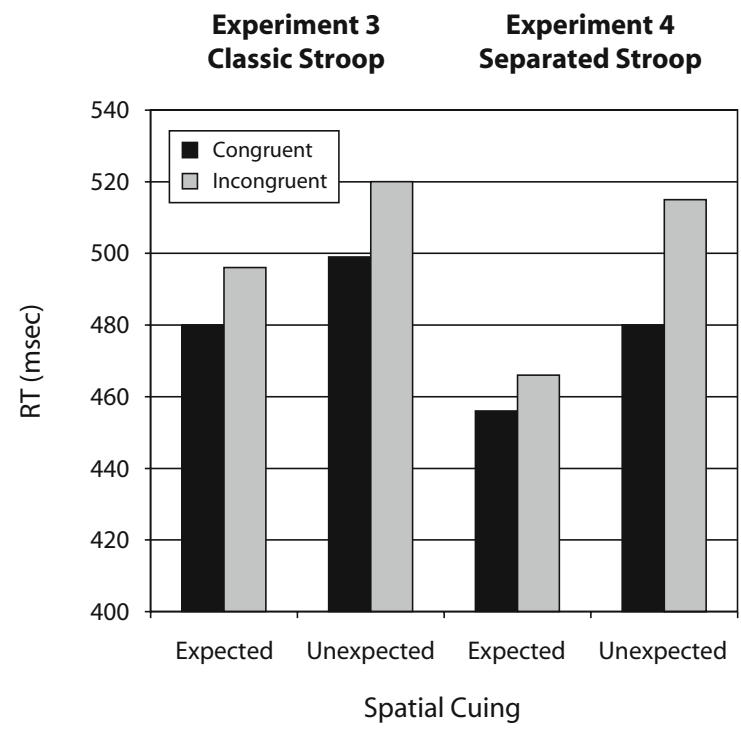

Figure 2. Posner and Stroop effects with integrated (Experiment 3) and spatially separated (Experiment 4) Stroop colorword stimuli. The differences in reaction time (RT) between expected and unexpected locations document the effects of spatial orientation of attention. The differences in RT between congruent and incongruent stimuli at each location document Stroop effects. Note that the Stroop effects are space invariant for integrated stimuli but are larger at unexpected than at expected locations with separated stimuli.

8.84, $p<.005]$. The 5 -msec difference between the two Stroop effects was not reliable $(F<1)$.

\section{Discussion}

The outcome of Experiment 3 is unmistakable: Both Posner and Stroop species of attention influenced performance, but the effects were additive. Notably, the Stroop effects observed at cued and miscued locations were roughly the same. This result reinforces those of Experiment 1 but with the classic Stroop stimuli (free from concerns of excessive difficulty). Recall that the Stroop task is a prime example of executive processing that entails the analysis of the stimulus onto its constituent dimensions. The Stroop effect actually gauges (the failure of) this selective decomposition. Our results show that this form of analytic attention is independent of that of spatial attention (gauged by Posner's orienting effect). We also note that the results of Experiment 3 replicate those reported by Shalev and Algom (2000) under similar (but not identical) conditions.

The question of consequence is this: Would the separability of the Posner and Stroop effects be observed in a condition in which the components of the Stroop stimulus are separated in space? Note that by divorcing the color from the word, spatial attention is imported to the realm of the Stroop task. Consequently, we predicted that, for spatially separated Stroop stimuli, spatial attention impacts color performance. Moreover, we predicted that, unlike with spatially integrated Stroop stimuli, the two systems of attention interact to shape performance with spatially separated Stroop stimuli.

\section{EXPERIMENT 4 \\ The Orientation of Attention to the Color of Spatially Separated Stroop Displays}

Experiment 4 mimicked Experiment 3, with a single notable exception: The display on each trial entailed a color word in black accompanied by a colored bar. The task for the participants remained the same as that in Experiment 3: to classify the color speedily and accurately. This arrangement is not new, of course, and appreciable Stroop effects have been demonstrated with the components separated in space (Cho, Lien, \& Proctor, 2006; Kahneman \& Chajczyk, 1983). What has been less largely recognized is that extending the Stroop stimulus in space might modify the nature of the task. The arrangement invites spatial attention to exert its influence where only analytic attention counted (with the classic integrated version). This influence has not been measured in the past (the centrally presented displays were certainly unsuitable), nor has the possible interaction with analytic attention been tested. We did both in Experiment 4. In particular, the comparison of the results in Experiments 3 and 4 is bound to provide converging evidence on these questions, as well as on the dissociation of the pertinent systems of attention.

\section{Method}

Participants. The same 16 participants from Experiment 3 took part in Experiment 4. In point of fact, half of the participants first performed Experiment 3 and half first performed Experiment 4 . The two experiments were separated by at least $3 \mathrm{~h}$ (and up to several days). The order of testing did not affect the results, nor did it interact with any of the other main effects ( $F \mathrm{~S}<1$ in all tests with order).

Stimuli,Apparatus, and Design. The apparatus and design were the same as those in Experiment 3. However, the display containing the Stroop stimulus differed. The color word was presented in black with a colored bar appearing below (on half of the trials) or above the word. The colored bar extended $3 \mathrm{~mm}$ in height and $15 \mathrm{~mm}$ in width, with the latter coinciding with the horizontal extent of the word. The bar and the word were presented in close vicinity (with a mere 5 pixels of vertical separation). This Stroop configuration was presented within one of two lateralized boxes. The size and placement of the boxes were the same as those in Experiment 3. The advance cue used was also the same as that in Experiment 3. The task for the participants was to identify the color of the bar as quickly as possible.

All of the other details of the experiment- the number of congruent and incongruent stimuli, the locations of the targets, the sequencing and timing of trials-were the same as in Experiment 3.

Procedure. The procedure was the same as in Experiment 3.

\section{Results}

The results appear in the right-hand panel of Figure 2. Both Posner and Stroop effects are discernible. For the former, performance was better in expected than in unexpected locations. The difference favoring valid orientation was appreciable, amounting to $36 \mathrm{msec}[F(1,15)=38.7$, $p<.0001]$. Also evident in Figure 2 are the Stroop effects in both expected and unexpected locations. The effect was $10 \mathrm{msec}$ when the stimulus appeared in an expected loca- 
tion $[F(1,15)=1.66, p=.10]$, but it was $35 \mathrm{msec}$ when the stimulus appeared in an unexpected location $[F(1,15)=$ $16.2, p<.001]$. The Stroop effect obtained with spatially unattended stimuli was thus three times the value of that obtained with spatially attended stimuli $[F(1,15)=6.63$, $p<.01]$.

\section{Discussion}

The outcome of Experiment 4 provides a clear answer to the question posed at the outset of the final pair of experiments of this study. In Experiment 4, the Posner and Stroop effects observed were not independent but, rather, interacted in determining performance. In particular, the Stroop effects for spatially attended and unattended stimuli differed appreciably. We attribute the interaction of the two modes of attention to the spatial separation of the stimulus components effected in Experiment 4. This separation exposed the Stroop stimulus to spatial processing in addition to that of analytic processing. Our manipulation rendered the Stroop stimulus somewhat similar to the flanker stimulus. The attention results support our predictions in that the two systems interacted in processing.

Considered in tandem with the results of Experiment 3, the present data provide strong support for the double dissociation accomplished in the present study. As we recounted, the Stroop task is the prime example of analytic attention, and this mode is impervious to spatial intrusions. We now add the caveat that this exclusive analysis holds only for the classic integrated Stroop stimuli. However, once the Stroop stimulus is separated in space, the Stroop effect still obtains, but it is modulated by spatial attention, too.

\section{GENERAL DISCUSSION}

Space and objects in space command visual attention, but so do stimulus attributes that inhere in the same objects and occupy the same spatial position. Our research encourages the idea that both species of visual attention are needed for adaptation and survival. Humans (and nonhumans) live in a complex perceptual world in which they must continually determine the most relevant regions or objects for their current activities. Just as critical, they must determine the most relevant attribute of the selected objects. After all, virtually all objects possess multiple attributes, of which only one (or a small subset) is relevant for current action. Does space- or object-based selection interact with attribute- or information-based selection?

In the present series of experiments, we explored the relationship between these two species of attention. The first system was gauged by Posner's orientation of attention design. The second system was gauged by Stroop stimuli and tasks. Notably, the two systems were embedded in a common experimental setup. The results revealed that the pertinent processes of attention are unrelated. However, when the Stroop task was replaced by the flanker task or when the Stroop stimuli were separated in space, the processes interacted. We believe that the root cause of the interaction is the spatial component inherent in the flanker and in the separated Stroop tasks. These tasks engage some of the same processes that the Posner task does-hence, the interaction. In summary, the present dissociation revealed space-based and information-based attention to be independent processes. Simultaneously, our data encourage vigilance when selecting tasks for the various systems of attention. Improper selection undermines the quest to specify the sought relationship.

The last point is notable. Ill-specified tasks carry costs for theoretical resolution. For example, we have already mentioned that Fan et al. (2002; see also Callejas et al., 2004, and Rueda et al., 2004) used the flanker task to represent the executive or intraobject system in their quest to isolate the three attentional networks suggested by Posner and his associates (the attention network test; Posner \& Petersen, 1990; Posner \& Raichle, 1994; Posner \& Rothbart, 2005). Contrary to their hypothesis and inconsistent with Posner's independent networks scheme, Fan et al. found that the flanker effect did depend on the spatial orientation of attention. In our interpretation, this surprising interaction (from the authors' point of view) was derived from the inappropriate choice of the flanker task to stand for analytic attention. Although the flanker task entails response congruity (or incongruity), a feature that it partially shares with the Stroop task, it also carries a spatial component, just like Posner's orientation of attention task. This latter component is crucial and engenders the observed interaction (see also Redick \& Engle, 2006).

Therefore, the independent networks of attention scheme advanced by Posner and his associates is well taken; what we take exception to is the insufficient resolution of the tasks that have been used to tap the different systems. Nevertheless, the present dissociation between spatial versus analytic attention does not fully overlap with the Posnerian dichotomy of orienting versus executive attention (see also MacDonald, Cohen, Stenger, \& Carter, 2000). Consider the first system of orientation of attention. The origin for orienting is in overt eye movements, and this mode of attention still carries the notion of focusing as its essence. The present notion of spatial attention emphasizes the selection aspect of this system: Spatial attention selects one among several well-defined, even salient, objects or regions in space. Now, consider the second Posnerian system of executive attention. The main function examined with respect to executive attention has been conflict resolution. The conflict often resides within a given object, but not necessarily so. This distinction has not been stressed in the pertinent research. In our scheme, the operation of analytic attention decomposes the object into its constituent dimensions, regardless of whether the dimensions conflict, agree, or are just neutral with respect to one another. So, the scope of analytic attention reaches beyond conflict resolution. Our system of analytic attention excludes any spatial involvement in this type of selection. Because the various attributes of the object reside in the same space (the color and the word in Stroop stimuli; the loudness, pitch, and timbre of a given voice), selection cannot be space or object based. This feature, too, supports the total dissociation of the two systems of attention. In sum, the current classi- 
fication of attention into spatial versus analytic systems is largely but not fully convergent with Posner's classification into orienting versus executive systems.

We conclude by stressing the need for a more inclusive and comprehensive view of human selective attention. Space- and object-based approaches, on one hand, and structure- or information-based approaches, on the other hand, are each capable of accounting for only a portion of routine human attention. The mechanism responsible for spatial and/or object selection is incapable, in principle, of performing the intraobject analyses that disentangle stimulus dimensions inherent in the same object at the same location. Conversely, this latter, information-based mechanism is not an ideal one for the selection of regions and objects in the visual field. Researchers from the two camps should not be myopic in their theoretical outlook. One is often left with the impression that processes of space and object selection exhaust visual selective attention. One can easily ignore the prior processes of object selection, as well, when the emphasis is on the isolation of information inhering in the attributes of the (single) object. In a sense, researchers from the first camp often leave the story of attention too early, whereas those from the second camp enter it a bit late. In everyday attention functioning, both processes are needed and executed on a routine basis.

Let us end with an instructive example from outside vision. Suppose that you wish to listen to an important voice message. One mechanism of attention is needed to select this voice from the multitude of sounds in the environment. Once this selection has been made, one must mentally isolate the semantic dimension of the target voice. After all, the physical sound possesses the attributes of loudness, pitch, timbre, volume, and density, apart from its meaning, which is the target dimension. Hence, a second mechanism, which is ideal for extracting information from a given physical signal, is needed and activated. The present results suggest that the two mechanisms are independent but that both are needed in normal mental functioning.

\section{AUTHOR NOTE}

We thank Rob Gordon, Lisa Fournier, and an anonymous reviewer for helpful comments on earlier versions of this article. We also thank Shay Ben-Haim for help in collecting the data. Correspondence concerning this article should be addressed to E. Chajut, Department of Psychology and Education, The Open University of Israel, Ra'anana 45677, Israel (e-mail: eranch@openu.ac.il).

\section{REFERENCES}

Algom, D., Dekel, A., \& Pansky, A. (1996). The perception of number from the separability of the stimulus: The Stroop effect revisited. Memory \& Cognition, 24, 557-572.

Botvinick, M. M., Carter, C. S., Braver, T. S., Barch, D. M., \& Cohen, J. D. (2001). Conflict monitoring and cognitive control. Psychological Review, 108, 624-652.

Callejas, A., Lupiáñez, J., \& Tudela, P. (2004). The three attentional networks: On their independence and interactions. Brain \& Cognition, 54, 225-227.

Chen, Z. (2003). Attentional focus, processing load, and Stroop interference. Perception \& Psychophysics, 65, 888-900.

Cho, Y. S., Lien, M. C., \& Proctor, R. W. (2006). Stroop dilution depends on the nature of the color carrier but not on its location. Journal of Experimental Psychology: Human Perception \& Performance, 32, 826-839.

Danziger, S., Kingstone, A., \& WARD, R. (2001). Environmentally defined frames of reference: Their time course and sensitivity to spatial cues and attention. Journal of Experimental Psychology: Human Perception \& Performance, 27, 494-503.

EriKsen, B. A., \& EriKsen, C. W. (1974). Effects of noise letters upon the identification of a target letter in a nonsearch task. Perception \& Psychophysics, 16, 143-149.

Eriksen, B. A., Eriksen, C. W., \& Hoffman, J. E. (1986). Recognition memory and attentional selection: Serial scanning is not enough. Journal of Experimental Psychology: Human Perception \& Performance, 12, 476-483.

Eriksen, C. W., \& Hoffman, J. E. (1973). The extent of processing of noise elements during selective encoding from visual displays. Perception \& Psychophysics, 14, 155-160.

ERIKSEN, C. W., \& ST. JAmes, J. D. (1986). Visual attention within and around the focus of attention: A zoom lens model. Perception \& Psychophysics, 40, 225-240.

Fan, J., McCandliss, B. D., Fossella, J., Flombaum, J. I., \& Posner, M. I. (2005). The activation of attentional networks. NeuroImage, 26, 471-479.

Fan, J., McCandliss, B. D., Sommer, T., Raz, A., \& Posner, M. I. (2002). Testing the efficiency and independence of attentional networks. Journal of Cognitive Neuroscience, 14, 340-347.

FolK, C. L., Remington, R. W., \& Johnston, J. C. (1992). Involuntary covert orienting is contingent on attentional control settings. Journal of Experimental Psychology: Human Perception \& Performance, 18, 1030-1044.

Goldsmith, M. (1998). What's in a location? Comparing object-based and space-based models of feature integration in visual search. Journal of Experimental Psychology: General, 127, 189-219.

Kahneman, D., \& ChaJCZYK, D. (1983). Tests of the automaticity of reading: Dilution of Stroop effects by color-irrelevant stimuli. Journal of Experimental Psychology: Human Perception \& Performance, 9, 497-509.

Kinchla, R. A., Solis-Macias, V., \& Hoffman, J. (1983). Attending to different levels of structure in a visual image. Perception \& Psychophysics, 33, 1-10.

Kunn, G., \& Kingstone, A. (2009). Look away! Eyes and arrows engage oculomotor responses automatically. Attention, Perception, \& Psychophysics, 71, 314-327.

LACHTER, J., Forster, K. I., \& RuthrufF, E. (2004). Forty-five years after Broadbent (1958): Still no identification without attention. Psychological Review, 111, 880-913.

LupiáñeZ, J., \& Funes, M. J. (2005). Peripheral spatial cues modulate spatial congruency effects: Analysing the "locus" of the cueing modulation. European Journal of Cognitive Psychology, 17, 727-752.

MacDonald, A. W., III, Cohen, J. D., Stenger, V. A., \& Carter, C. S. (2000). Dissociating the role of the dorsolateral prefrontal and anterior cingulate cortex in cognitive control. Science, 288, 1835-1838.

MacLeod, C. M. (1991). Half a century of research on the Stroop effect: An integrative review. Psychological Bulletin, 109, 163-203.

Martin, M. (1979). Local and global processing: The role of sparsity. Memory \& Cognition, 7, 476-484.

Melara, R. D., \& Algom, D. (2003). Driven by information: A tectonic theory of Stroop effects. Psychological Review, 110, 422-471.

MelarA, R. D., \& Mounts, J. R. W. (1993). Selective attention to Stroop dimensions: Effects of baseline discriminability, response mode, and practice. Memory \& Cognition, 21, 627-645.

NAVON, D. (1977). Forest before trees: The precedence of global features in visual perception. Cognitive Psychology, 9, 353-383.

PomerantZ, J. R. (1983). Global and local precedence: Selective attention in form and motion perception. Journal of Experimental Psychology: General, 112, 516-540.

Posner, M. I. (1978). Chronometric explorations of the mind. Hillsdale, NJ: Erlbaum.

Posner, M. I. (1980). Orienting of attention. Quarterly Journal of Experimental Psychology, 32, 3-25.

Posner, M. I., \& Cohen, Y. (1984). Components of visual orienting. In H. Bouma \& D. G. Bouwhuis (Eds.), Attention and performance X: Control of language processes (pp. 531-556). Hillsdale, NJ: Erlbaum. 
Posner, M. I., \& Petersen, S. E. (1990). The attention system of the human brain. Annual Review of Neuroscience, 13, 25-42.

Posner, M. I., \& Raichle, M. E. (Eds.) (1994). Images of mind. New York: Scientific American Library.

Posner, M. I., \& Rothbart, M. K. (2004). Hebb's neural networks support the integration of psychological science. Canadian Psychology, 45, 265-278.

PosNer, M. I., \& RothbaRT, M. K. (2005). Influencing brain networks: Implications for education. Trends in Cognitive Sciences, 9, 99-103.

Posner, M. I., SNyder, C. R. R., \& Davidson, B. J. (1980). Attention and the detection of signals. Journal of Experimental Psychology: General, 109, 160-174.

Redick, T. S., \& ENGLE, R. W. (2006). Working memory capacity and attention network test performance. Applied Cognitive Psychology, 20, 713-721.

Rueda, M. R., Fan, J., Halparin, J., Gruber, D., Lercari, L. P., McCANDliss, B. D., \& Posner, M. I. (2004). Development of attention during childhood. Neuropsychologia, 42, 1029-1040.

Shalev, L., \& Algom, D. (2000). Stroop and Garner effects in and out of Posner's beam: Reconciling two conceptions of selective attention. Journal of Experimental Psychology: Human Perception \& Performance, 26, 997-1017.

Stoffer, T. H. (1993). The time course of attentional zooming: A comparison of voluntary and involuntary allocation of attention to the levels of compound stimuli. Psychological Research, 56, 14-25.

STRoop, J. R. (1935). Studies of interference in serial-verbal reaction. Journal of Experimental Psychology, 18, 643-662.

WARD, L. M. (1982). Determinants of attention to local and global fea- tures of visual forms. Journal of Experimental Psychology: Human Perception \& Performance, 8, 562-581.

\section{NOTES}

1. The Stroop effect (SE) is often subdivided into Stroop interference (SI) and Stroop facilitation (SF), when neutral stimuli (N) such as the word table printed in a color are also presented. In terms of reaction time (RT), the Stroop effect is the difference between RTs for color naming for incongruent (IC) and congruent (C) stimuli [SE = RT(IC) - RT(C)]. Stroop interference is then defined as SI $=\mathrm{RT}(\mathrm{IC})-\mathrm{RT}(\mathrm{N})$, and Stroop facilitation as $\mathrm{SF}=\mathrm{RT}(\mathrm{N})-\mathrm{RT}(\mathrm{C})$. Clearly, too, $\mathrm{SE}=\mathrm{SI}+\mathrm{SF}$.

2. Several features of Chen's (2003) study preclude drawing firm conclusions. First, Chen presented the target words for a very long duration, thereby permitting eye movements rather than visual attention per se to influence performance. Second, Chen did not present congruent stimuli; consequently, she did not derive the Stroop effect, but derived only Stroop interference (the difference between performance for incongruent and neutral stimuli). Finally, a hidden correlation over the experimental trials between word and color was present in the design. Noticing the irrelevant word first, the participants had a better-than-chance probability of guessing the target color. Thus, the nominally irrelevant word became informative of the color. Because there were (many) more valid than invalid trials, this information was especially poignant with the former, which thus spuriously enhanced the Stroop effect.

(Manuscript received April 16, 2008; revision accepted for publication March 12, 2009.) 